\title{
DIETA DA GURIJUBA - SCIADES PARKERI (TRAILL 1832) - CAPTURADA PELAS PESCARIAS ARTESANAIS DE LARGA ESCALA DA COSTA NORTE DO BRASIL
}

\author{
DUARTE-PAULA, J. ${ }^{* *}$; NOGUEIRA, L.C. ${ }^{\text {; }}$ NUNES, Z.M.P. ${ }^{2} \&$ BENTES,B. ${ }^{1}$ \\ 1. Laboratório de Bioecologia Pesqueira, Instituto de Estudos Costeiros (IECOS), Universidade \\ Federal do Pará (UFPA), Bragança, PA, Brasil. \\ 2. Laboratório de Qualidade de Água, Instituto de Estudos Costeiros (IECOS), Universidade Federal \\ do Pará (UFPA), Bragança, PA, Brasil. \\ *Corresponding author: jeannepd@gmail.com
}

\begin{abstract}
Duarte-paula, J.; Nogueira, L.C.; Nunes, Z.M.P. \& Bentes, B., (2016) . Dieta da Gurijuba - Sciades parkeri (traill 1832) - capturada pelas pescarias artesanais de larga escala da costa norte do Brasil. Braz. J. Aquat. Sci. Technol. 20(1). eISSN 1983-9057. DOI: 10.14210/bjast.v20n1. Sciades parkeri, is a marine /estuarine fish, popularly known as Gillbacker Sea Catfish. Was studied the diet and bimonthly sampling were collected in the Amapá coast, between September 2011 to December 2012. In laboratory, the stomachs were removed, weighted and their gastric contents were analyzed using stereomicroscopy. Diet was assessed by frequency of occurrence methods, volumetric analysis index and feeding importance index. A total of 189 stomachs were analyzed; of these, 139 contained food and 25 were empty. Main food items were crustaceans fragments $(n=60)$, fish $(n=33)$ and Cycloplax pinnotheroides (Brachyura) $(n=22)$. Items that have the highest frequencies of occurrence and feeding importance index in the rainy and dry season was crustaceans fragments (rainy Fo $=38.64 \%$, Ali $=65.35 \%$ and in dry season Fo $=48,15 \%$ e Ali $=67,76 \%$ ) and fish fragments (rainy Fo $=20.45 \%$, $\mathrm{Ali}=19.89 \%$ and in dry season $\mathrm{Fo}=27,78 \%$ e Ali $=23,45 \%$ ). Analyses indicated that $\mathrm{S}$. parkeri is essentially carnivorous.
\end{abstract}

Keywords: Feed ecology, Fish diet, Ariidae.

\section{INTRODUÇÃO}

Diversos peixes, ao longo do ciclo de vida, passam por alteração nos padrões de alimentação que geralmente estão relacionados a fatores internos (biologia, comportamento e fisiologia) e externos (habitats, disponibilidade de recursos, risco à predação e competição), que fazem com que desenvolvam estratégias para a otimização do alimento disponível (Luczkoviche, 2001; Abelha et al., 2001).

Muitos organismos possuem a estratégia de modificar o uso dos recursos alimentares de acordo com a oferta disponibilizada no ambiente (Stephens \& Krebs, 1986), que é constantemente influenciada pelas mudanças temporais (Hammerschlag et al., 2010). Desta maneira, o uso do recurso está relacionado a diversidade dos itens da região (Layman et al., 2007).

Neste sentido, o estudo sobre a ecologia alimentar do conteúdo estomacal tem sido uma ferramenta importante para a compreensão das relações ecológicas e tróficas das comunidades aquáticas, por tentar compreender a relação entre presa e predador (Layman et al., 2007; Hammerschlag et al., 2010). Além disso, é uma importante ferramenta para definição de estratégias de manejo adequadas aos recursos pesqueiros ali encontrados (Helfman,1999).

A grande oferta de alimento disponível à fauna bentônica e demersal na zona costeira, perfaz um ambiente propício às altas densidades de espécies ao longo da pluma do rio Amazonas e a costa leste do Pará (Viana et al., 2010). Dentre as espécies que estão inseridas na região destaca-se Sciades parkeri (Traill 1832), um bagre marinho/estuarino de hábito bentônico (Marceniuk, 2005), cuja biologia e a dinâmica populacional ainda são pouco conhecidas.

Apesar dos poucos estudos direcionados a $S$. parkeri, localmente conhecida como gurijuba, existe um sistema pesqueiro especializado para a sua captura (Bentes et al., 2012) em decorrência da boa aceitação de sua carne no mercado local e nacional (Nascimento et al., 2002). O sistema pesqueiro da gurijuba é formado por frotas artesanais de pequena e média escala, que utilizam diversas artes de pesca como as redes de emalhar e espinheis de anzóis. Os desembarques são constantes e ocorrem durante todo o ano (MMA, 2006).

O aumento gradativo do esforço pesqueiro empregado na captura da gurijuba e a ausência de medidas eficazes de ordenamento fez com que a espécie entrasse na lista vermelha da IUCN (International Union for Conservation of Nature and Natural Resources, versão 2014.3) sendo classificada como uma espécie vulnerável. A primeira tentativa de gerenciamento, foi o decreto Lei ${ }^{\circ} 221$ IBAMA/MMA), que proíbe, no estado do Amapá, a pesca da espécie no período de $1^{\circ}$ de novembro a 31 de março na área 
de desembocaduras dos Rios Araguari e Cunani, até o limite de três milhas náuticas e entorno das ilhas de Macará e Jipipoca. Esta mesma portaria permite a pesca da espécie mesmo durante o período estabelecido como defeso ( $1^{\circ}$ de novembro a 31 de março), desde que as artes de pesca utilizadas sejam linha, anzol /ou espinhel.

Mesmo com as incertezas dos dados de esforço e produção, a diminuição da densidade dos estoques de S. parkeri demonstrado nas capturas é evidente, a julgar pelos volumes desembarcados nos principais pólos pesqueiros do estado do Pará e Amapá (Pinheiro e Frédou, 2004; MPA, 2006).

Neste contexto, a ausência de estudos que abordem a composição detalhada e utilização dos recursos alimentares de $S$. parkeri nos mostra a necessidade de se obter informações biológicas básicas que subsidiem propostas de manejo mais abrangentes para a espécie, tendo em vista seu valor comercial para a pesca artesanal na região costeira amazônica. Por isso, o objetivo deste trabalho foi caracterizar a dieta de S. parkeri capturada nas pescarias comerciais da costa Norte do Brasil.

\section{MATERIAL E MÉTODOS}

\section{Área de Estudo}

A costa Norte brasileira inclui os Estados do Amapá, Pará e Maranhão, e é uma região dominada por macromarés influenciadas pelas correntes Norte do Brasil e Sul Equatorial, cujas ondas mais frequentes são perpendiculares à costa. As planícies de mangue dominam toda a área, seguidas pelas planícies costeiras que alargam em direção ao Cabo Orange, estreitam ao centro e novamente alargam em direção à costa do Pará (MMA, 2008).

A plataforma continental tem largura compreendida entre 80 e 160 milhas náuticas e é recoberta por depósitos lamosos que favorecem a exploração de recursos pesqueiros que habitam esses substratos. Em alguns locais, os sedimentos lançados pela vazão do Rio Amazonas (silte e argila) alcançam o oceano, formando pequenas falésias que caracterizam o golfão amazônico (MMA, 2006).

Grande parte da matéria orgânica desta área é proveniente da decomposição das florestas de mangue e pelas planícies inundadas do rio Amazonas, que fornecem condições ideais para uma elevada produtividade pesqueira (Isaac, 2006; Barthem \& Fabré, 2004).

\section{Método de amostragem}

Foram realizadas coletas bimestrais, entre setembro de 2011 e dezembro de 2012 junto à frota comercial atuante na Costa do Pará e Amapá. Os indivíduos foram selecionados aleatoriamente de forma a representar pelo menos $1 / 3$ do volume total de $S$. parkeri da produção da embarcação.

As capturas ocorreram com espinhel de fundo e anzol $n^{\circ} 13$, iscados geralmente com sardinhas (que não foram incluídas nas análises de conteúdos estomacais). O cabo principal do espinhel era de $6.000 \mathrm{~m}$ com aproximadamente 3.000 anzóis, separados por linhas secundárias que se distanciavam cerca de $2 \mathrm{~m}$ uma da outra. As viagens tiveram duração média de 20 dias e os indivíduos foram selecionados por lance (cerca de 40 lances por viagem), que eram realizados duas vezes ao dia com uma duração média de sete horas e meia cada um. Os locais de amostragem estão apresentados na figura 1.

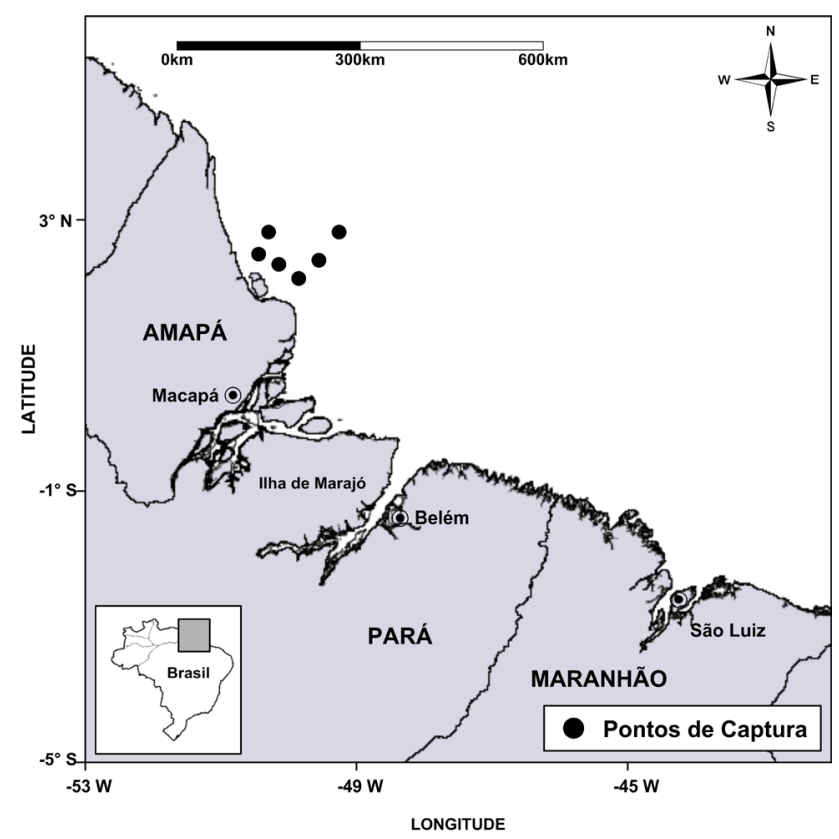

Figura 1- Pontos de coleta de Sciades parkeri realizada entre setembro de 2011 e dezembro de 2012 na costa Norte do Brasil.

Todos os espécimes selecionados aleatoriamente, receberam injeções de formol a $10 \%$ na cavidade visceral imediatamente após a captura. Depois, foram acondicionados na urna da embarcação contendo gelo, até serem desembarcados no trapiche da cidade de Bragança, no Estado do Pará, para serem desembarcadas e transportados em caixas térmicas com gelo ao laboratório de Bioecologia Pesqueira da Universidade Federal do Pará.

Os períodos sazonais foram estabelecidos pelos volumes totais de chuva a partir dos dados do Instituto Nacional de Meteorologia (INMET). Assim, o período chuvoso compreendeu os meses de janeiro a julho e o período seco de agosto a dezembro (Figura 2). 


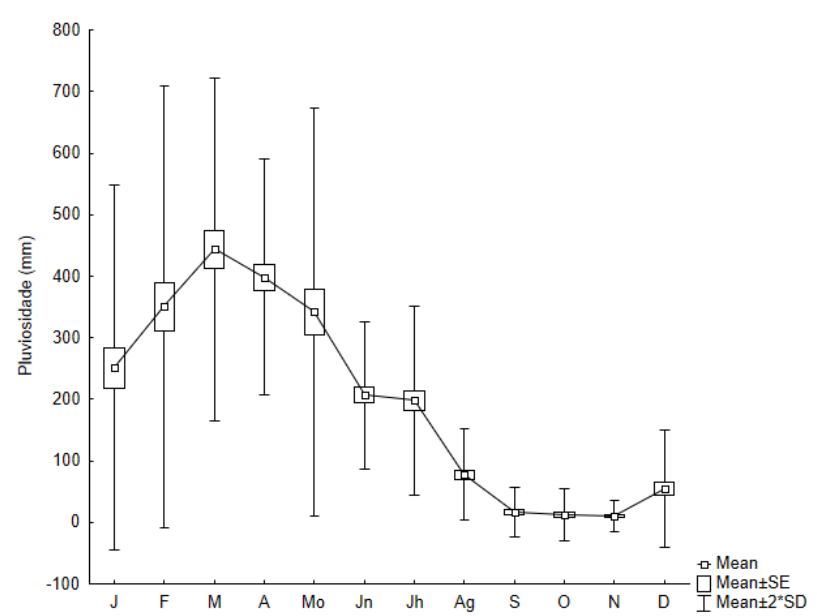

Figura 2 - Pluviosidade mensal (média, erro e desvio padrão) da estação meteorológica de Macapá-AP no período de janeiro de 1993 a dezembro de 2012.

No laboratório, os exemplares tiveram sua identificação confirmada de acordo com Marceniuk (2005) e registradas as seguintes medidas: comprimento total $(\mathrm{CT}$ em $\mathrm{cm})$ comprimento padrão $(\mathrm{CP}$ em $\mathrm{cm})$ e peso total (PT em g).

Posteriormente, efetuou-se uma incisão longitudinal da abertura anal até a altura da inserção das nadadeiras pélvicas para identificação das gônadas, afim de identificar macroscopicamente os sexos, de acordo com metodologia proposta por Vazzoler (1996), onde as fêmeas foram classificadas em cinco estágios de maturação gonadal ( $A$ - imaturo, $B$ - em maturação; $C$ - maturo e $D=$ desovado) e os machos em dois ( $A$ - imaturo e $C$ - maturo). Logo em seguida foi realizada a retirada dos estômagos para as análises de seu conteúdo.

\section{Estudo da dieta}

Do total de espécimes capturados de S.parkeri, apenas 189 foram usados para análise de conteúdo estomacal, pois foram retirados os indivíduos de sexo indeterminado, uma vez que um dos fatores testados nas análise foi a variação da dieta por sexo.

Após a retirada dos estômagos, estes foram lavados com água destilada, pesados, e o volume foi obtido por deslocamento de líquido utilizando-se uma bateria de provetas graduadas. Os estômagos foram seccionados e os itens alimentares foram examinados com o auxílio de um estereomicroscópio, identificados de acordo com a literatura disponível (Figuereido \& Menezes 1980; Cervigón et al., 1993; Melo, 1996), e quantificados. Também foram realizadas consultas a pesquisadores especializados na identificação para se chegar ao menor nível taxonômico por item (Hynes, 1950).

Os dados de conteúdo estomacal foram analisados de acordo com a metodologia proposta por Lima-Junior \& Goitein (2001) para obtenção da frequência de ocorrência, através da fórmula $F o=100$ $\mathrm{x}$ ni $\div$ n., onde Fo $=$ frequência de ocorrência do item alimentar "i" na amostra, ni = número de estômagos onde o "i" foi encontrado e $\mathrm{n}=\mathrm{o}$ número de estômagos com alimento; índice de análise volumétrica $(\mathrm{VI})$, dado pela fórmula $\mathrm{Vi}=25 \times \mathrm{Mi}$, onde: $\mathrm{Vi}=$ Índice de Análise Volumétrica; 25 = constante; $\mathrm{Mi}=$ média dos pontos atribuídos ao item alimentar "i"; e o índice de Importância Alimentar por meio da fórmula Ali = Fo $\mathrm{x} \mathrm{Vi}$, onde Ali = Índice de importância do alimento "i" na amostra; Fo = Frequência de ocorrência do item alimentar "i" na amostr e Vi = Índice de análise volumétrica.

\section{Análise de dados}

Para as análises foi utilizado o programa STATISTICA 7.0 (Statsoft INC.,2007). O comprimento padrão (CP em $\mathrm{cm})$, o peso total da espécie (PT), o peso do estômago cheio (PEC) e o peso do item (PI) foram todos registrados em grama e o volume do estômago (Vol) em $\mathrm{mL}$. A frequência de ocorrência (Fo), o índice de análise volumétrica e o índice de importância alimentar (Ali) foram testadas com ANOVA one way com erro de $5 \%$ considerando as premissas da análise (homocedasticidade e normalidade) entre estações (período chuvoso e seco) e sexo do indivíduo (macho e fêmea). Também foram realizados testes de médias de comprimento e biomassa (ANOVA; com erro de $5 \%$ ) entre os meses e os resultados foram plotados em gráficos com os respectivos intervalos de confiança das médias.

Para a análise dos itens alimentares, Índice de importância alimentar em relação ao comprimento, sexo e estação, os indivíduos foram agrupados, arbitrariamente, nas seguintes classes de comprimento padrão (em cm): CL1:<35; CL2: 35 à 40; CL3: 40 à 45; CL4: 45 à 50; CL: 50 à 55; CL6: 55 à 60 e CL7: $>60 \mathrm{~cm}$. Adicionalmente, os itens encontrados nos estômagos foram organizados em quatro categorias: (i) peixes, (ii) crustáceos e (iii) fragmentos de peixes e (iv) crustáceos, sendo estas duas últimas incluindo os itens que se encontravam em avançado estado de digestão, que impediam a identificação precisa.

\section{RESULTADOS}

No total, foram analisados 209 espécimes de S. parkeri dos quais 108 eram fêmeas, 54 machos e 47 indivíduos nos quais o sexo não foi identificado. As fêmeas foram predominantes durante todos os meses de coleta, sendo que no mês de março de 2012 as mesmas ocorreram em maior número que os machos.

A variação de tamanho e de peso foi observada 
durante todo o período da coleta com indivíduos de comprimento padrão médio de $46,55 \mathrm{~cm} \pm 3,49 \mathrm{~cm}$ e com peso médio de $1706,05 \mathrm{~g} \pm 368 \mathrm{~g}$ (Figura $3 \mathrm{~A} \mathrm{e}$ $3 \mathrm{~B})$. Os maiores indivíduos tanto em tamanho, quanto em peso foram capturados no mês de março, seguido pelo mês de agosto (Figura 3A e Figura 3B).
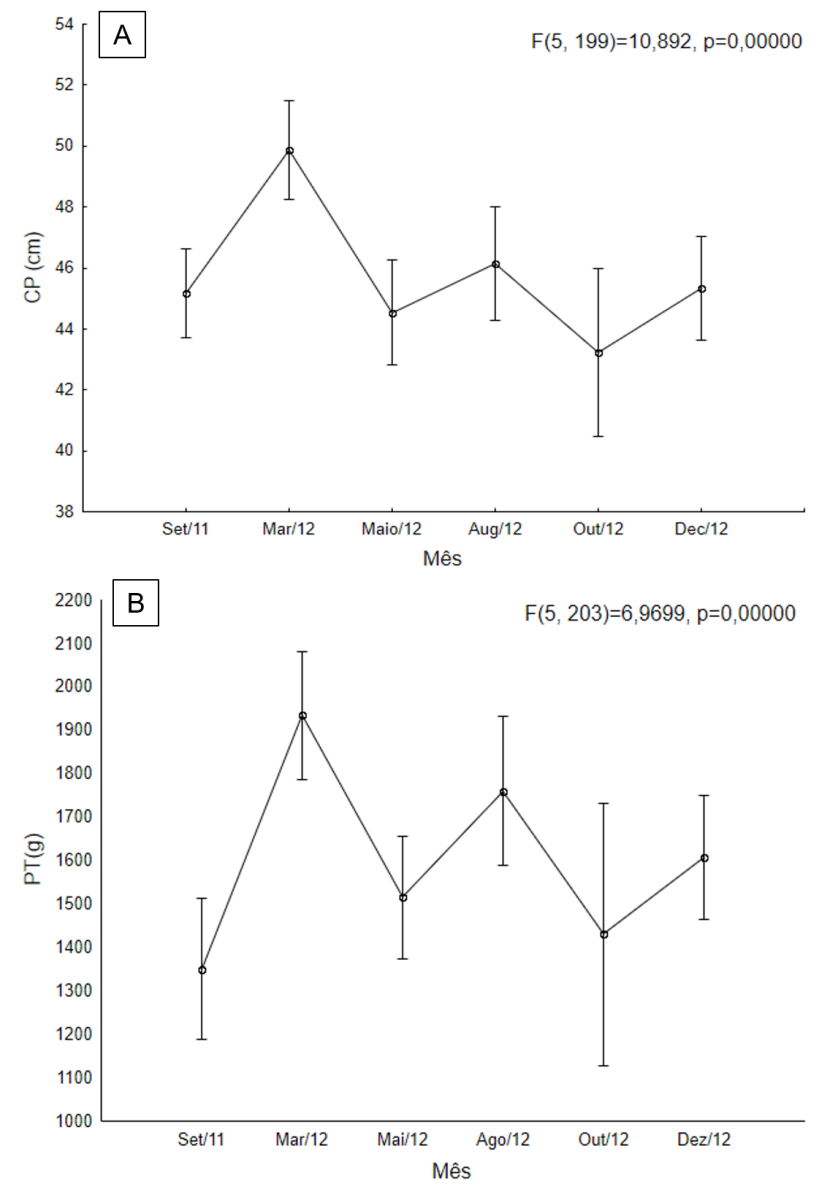

Figura 3- Variação da média e intervalo de confiança do comprimento padrão $(\mathrm{cm})$ e peso $(\mathrm{g})$ por mês de coleta (setembro/2011 a dezembro/2012) de S. parkeri capturados na costa Norte do Brasil. $(\mathrm{CP}=$ Comprimento padrão; $\mathrm{PT}=\mathrm{Peso}) . \mathrm{A}=$ variação mensal do comprimento padrão $(\mathrm{cm})$; B = variação mensal do peso $(\mathrm{g})$.

Durante todo o período de estudo, as fêmeas foram mais frequentes principalmente na classe de tamanho CL4 (48,08\% indivíduos) e os machos foram mais frequentes na classe de tamanho de CL3 e CL4 (ambos com 40,38\% indivíduos) (Figura 3). No período chuvoso, tanto machos quanto fêmeas foram mais abundantes na classe de tamanho CL4 $(47,45 \%$ e $43,75 \%$ indivíduos, respectivamente) (Figura 4).
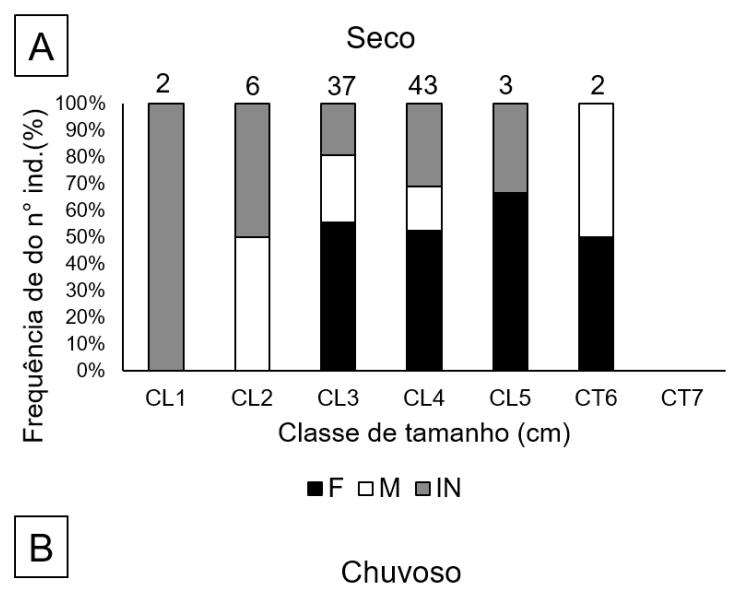

Chuvoso

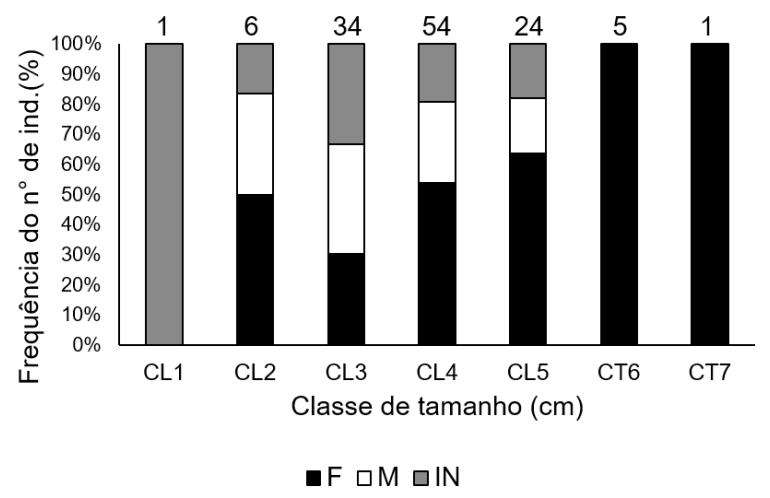

Figura 4 - Frequência de ocorrência dos indivíduos de S. parkeri por categoria de tamanho e sexo entre setembro/2011 a dezembro/2012 capturados na costa Norte do Brasil. A = período seco; B = período chuvoso. $\mathrm{F}$ = fêmeas; $\mathrm{M}=$ machos; $\mathrm{IN}=$ sexo indeterminado. Classes de tamanho: CL1:<35 cm; CL2: 35 a $40 \mathrm{~cm}$; CL3: 40 a $45 \mathrm{~cm}$; CL4: 45 a $50 \mathrm{~cm}$; CL: 50 a $55 \mathrm{~cm}$; CL6: 55 a $60 \mathrm{~cm}$ e CL7: $>60 \mathrm{~cm}$.

A frequência de indivíduos imaturos, tanto machos como fêmeas foi constante. Nos meses de março, maio e dezembro de 2012 houve maior número de fêmeas imaturas (17, 18 e 20 indivíduos, respectivamente), apenas no mês de março foi encontrado uma fêmea madura sexualmente (Figura 5A). As maiores ocorrências de machos imaturos foram nos meses de março e maio de 2012 (17 e 18 indivíduos, respectivamente), em contrapartida, machos maduros foram encontrados nos meses de setembro de 2011, março e dezembro de 2012 (3, 2 e 3 indivíduos, respectivamente) (Figura 5B).

A partir dos 189 estômagos de S. parkeri, 139 $(73,54 \%)$ continham alimentos, $25(13,23 \%)$ continham sedimentos (material lamoso encontrado nos estômagos em alto grau de decomposição no qual não foi possível identificar nenhum item alimentar) e 25 $(13,23 \%)$ estavam vazios (tabela 1$)$. Tanto no período chuvoso quanto no seco, os itens que apresentaram maior frequência de ocorrência e índice de importância alimentar (Ali) na dieta de S.parkeri foram os fragmentos de crustáceos (Chuvoso Fo= 38,64\%, Ali= 65,35\% e Seco Fo $=48,15$ e Ali= 67,76\%, (Tabela 1). 

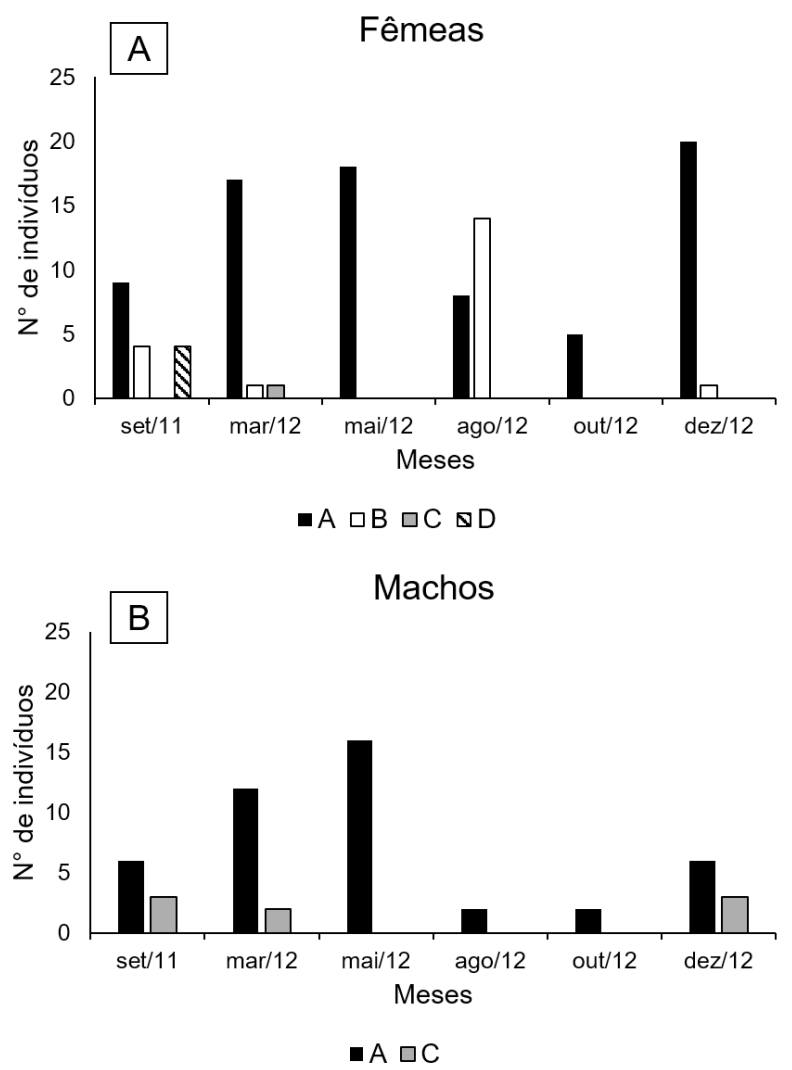

Figura 5 - Variação dos estágios gonadais de S. parkeri por mês de coleta entre setembro/2011 a dezembro/2012 capturados na costa Norte do Brasil. $A=$ imaturo; $B=$ em maturação; $C=$ maturo e $\mathrm{D}=$ desovado.

Tabela 1- Itens encontrados em 139 estômagos de Sciades parkeri capturados durante o período de setembro de 2011 a dezembro de 2012 pela frota comercial atuante na Costa do Pará e Amapá. Fo = frequência de ocorrência, $\mathrm{Vi}$ = Índice de análise volumétrica, Ali = índice de importância alimentar, FC: Fragmentos de camarão; FP: Fragmentos de peixe.

\begin{tabular}{l|ccc|ccc}
\hline & \multicolumn{7}{c}{ Chuvoso } & \multicolumn{3}{c}{ Seco } \\
Itens alimentares & Fo(\%) & V $_{i}(\%)$ & (Ali\%) & Fo(\%) & $V_{i}(\%)$ & (Ali\%) \\
\hline \multicolumn{7}{c}{ CRUSTACEA } \\
\hline \multicolumn{7}{c}{ Brachyura } \\
\hline Cycloplax pinnotheroides & 14,77 & 11,61 & 8,12 & 16,67 & 16,67 & 8,04 \\
Callinectes danae & - & - & - & 5,56 & 4,17 & 0,67 \\
Panopeus sp & 2,27 & 1,79 & 0,19 & - & - & - \\
\hline \multicolumn{7}{c}{ Penaeidae } \\
\hline Xiphopenaeus kroyeri & 4,55 & 3,57 & 0,77 & - & - & - \\
Penaeidae spp & 2,27 & 3,57 & 0,38 & - & - & - \\
F.C. & 38,64 & 35,71 & 65,35 & 48,15 & 48,61 & 67,76 \\
\hline \multicolumn{7}{c}{ OSTEICHTHYES } \\
\hline \multicolumn{7}{c}{ Sciaenidae } \\
\hline Macrodon ancylodon & 6,82 & 9,82 & 3,17 & 1,85 & 1,39 & 0,07 \\
Sciaenidae spp & 3,41 & 6,25 & 1,01 & - & - & - \\
Stellifer sp & 4,55 & 4,46 & 0,96 & - & - & - \\
\hline \multicolumn{7}{c}{ Ariidae } \\
\hline Ariidae spp & 1,14 & 1,79 & 0,1 & - & - & - \\
Cathorops spp & 1,14 & 0,89 & 0,05 & - & - & - \\
F.P. & 20,45 & 20,54 & 19,89 & 27,78 & 29,17 & 23,45 \\
\hline
\end{tabular}

O volume do estômago diferiu entre estações (com maior média no período chuvoso: $F=34,4$; $p<0,01$ ) e entre sexos (maior nos machos: $F=21,46$; $\mathrm{p}<0,01$ ) (Tabela 2).

A dieta de $S$. parkeri é constituída principalmente por crustáceos, que estiveram presentes em
Tabela 2- Resultados de ANOVA one way do Comprimento padrão (CP-cm), Peso total (PT - g), Peso do estômago cheio (PEC-g), Peso do conteúdo $(\mathrm{g})$, Volume do estômago $(\mathrm{ml})$, Peso do item $(\mathrm{g})$ e doíndice de importância alimentar (Ali) em função dos períodos sazonais (Chuvoso $=\mathrm{CH}$ e Seco $=\mathrm{S}$ ) e sexo (Fêmea=F; e Macho = M), de S. parkeri capturados de setembro de 2011 a dezembro de 2012 pela frota comercial atuante na Costa do Pará e Amapá. $\mathrm{F}=$ teste de Fisher; $\mathrm{P}=$ probabilidade.

\begin{tabular}{ccccc}
\hline Variável dependente & $\begin{array}{c}\text { Fator de } \\
\text { variação }\end{array}$ & $\mathbf{F}$ & $\mathbf{P}$ & $\begin{array}{c}\text { Interpretação } \\
\text { estatística * } \\
\text { gráfica }\end{array}$ \\
\hline CP (cm) & $\begin{array}{c}\text { Estação } \\
\text { Sexo }\end{array}$ & $\begin{array}{c}21,74 \\
0,53\end{array}$ & $<0,01$ & $\begin{array}{c}\mathrm{CH}>\mathrm{S} \\
\mathrm{F}>\mathrm{M}\end{array}$ \\
\hline PT (g) & Estação & 19,65 & $<0,01$ & $\mathrm{CH}>\mathrm{S}$ \\
Sexo & 13,01 & $<0,01$ & $\mathrm{~F}>\mathrm{M}$ \\
\hline PEC (g) & Estação & 1 & $>0,05$ & $\mathrm{CH}>\mathrm{S}$ \\
& Sexo & 9,84 & $<0,05$ & $\mathrm{M}>\mathrm{F}$ \\
\hline \multirow{2}{*}{ Vol. Estômago (m) } & Estação & 34,4 & $<0,01$ & $\mathrm{CH}>\mathrm{S}$ \\
& Sexo & 21,46 & $<0,01$ & $\mathrm{M}>\mathrm{F}$ \\
\hline \multirow{2}{*}{ Peso do item (g) } & Estação & 0,61 & $>0,05$ & $\mathrm{CH}>\mathrm{SH}$ \\
& Sexo & 0,82 & $>0,05$ & $\mathrm{~F}>\mathrm{M}$ \\
\hline \multirow{2}{*}{ Ali } & Sexo & 19,87 & $<0,01^{*}$ & $\mathrm{~F}>\mathrm{M}$ \\
\hline *se p<5\%. & Estação & 52,25 & $<0,01^{*}$ & $\mathrm{~S}>\mathrm{CH}$ \\
& Sexo & 20,50 & $<0,01^{*}$ & $\mathrm{~F}>\mathrm{M}$ \\
\hline & & & &
\end{tabular}

quase todas as classes de tamanho durante o período de amostragem. Nos estágios iniciais de maturação sexual, tanto as fêmeas quanto os machos apresentaram uma dieta diversificada e semelhante nos indivíduos imaturos e à medida que amadurecem sexualmente ocorre uma diminuição do espectro alimentar (Figura 6).

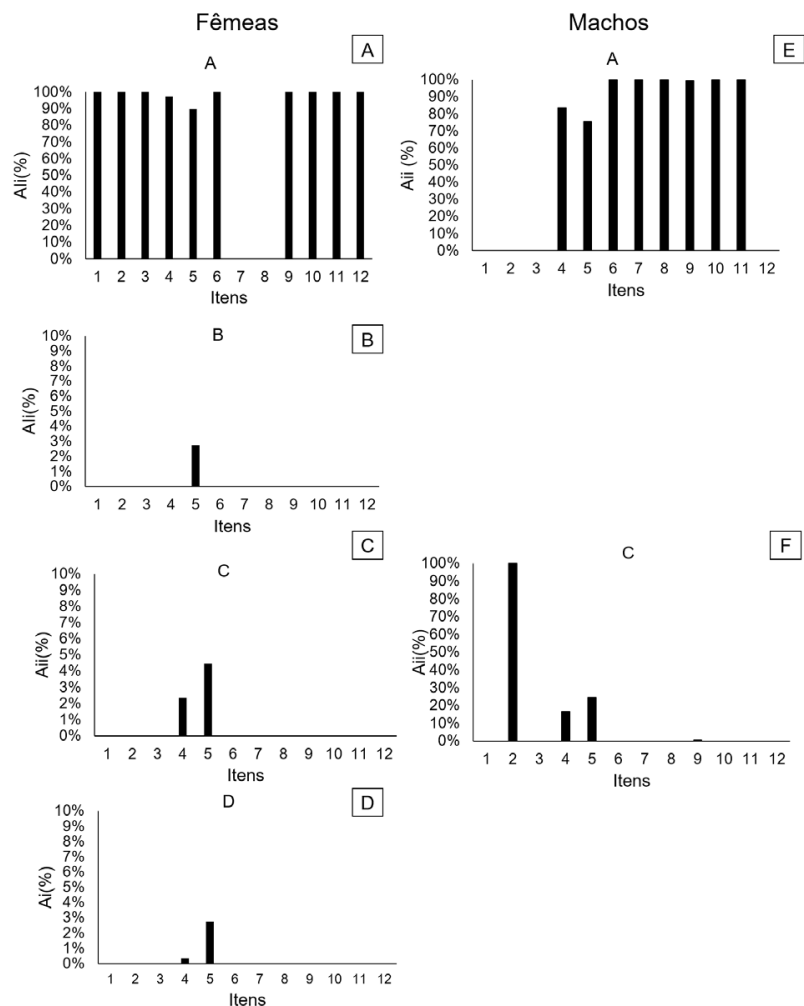

Figura 6 - Índice de importância alimentar por estágios de maturação gonadal de espécimes de $S$. parkeri capturados de setembro de 2011 a dezembro de 2012 na costa Norte do Brasil. A = imaturo; $\mathrm{B}=$ em maturação; $\mathrm{C}=$ maturo; $\mathrm{D}=$ desovado. 1 = Ariidae spp; 2 = Callinectes danae; 3 = Cathorops sp; 4 = Cycloplax pinnotheroides; 5 = fragmentos de crustáceos; 6 = fragmentos de peixes; 7 = Macrodon ancylodon; $8=$ Panopeus sp; 9 = Penaeidae spp 10 = Sciaenidae spp; 11 = Stellifer sp.; 12 = Xiphopenaeus kroyeri. 
No período chuvoso, o item mais importante (Ali\%) na dieta das fêmeas de S. parkeri, na classe de tamanho CL3 e CL4 foi principalmente espécimes de C. pinnotheroides $(67,56 \%$ e $62,61 \%$, respectivamente) seguido de fragmentos de crustáceos $(27,32 \%$ e $18,64 \%$, respectivamente) (Figura 7). Na classe CL5, os itens mais importantes na alimentação, foram C. pinnotheroides e fragmentos de peixes $(90,96 \%$ e $9,04 \%$, respectivamente) (Figura 7 ).

O principal item alimentar dos machos de S. parkeri na classe de tamanho CL2, durante a es- tação chuvosa, foi Penaeidae spp (100\%) (Figura 7). $\mathrm{Na}$ classe CL4, a dieta apresentou maior diversidade de alimentos (Figura 7).

Na estação seca o item mais importante (Ali\%) na dieta de S. parkeri, para as fêmeas, nas classes de tamanho CL3 e CL4 foram principalmente fragmentos de crustáceos e fragmentos de peixes $(85,13 \%$ e $58,03 \%$, respectivamente) (Figura 7 ). Para os machos pertencentes às classes de tamanho CL2, CL3, CL4 e CL5 foram fragmentos de crustáceos $(99,44 \%$; 76,99\%;100\% e 100\%, respectivamente) (Figura 7).

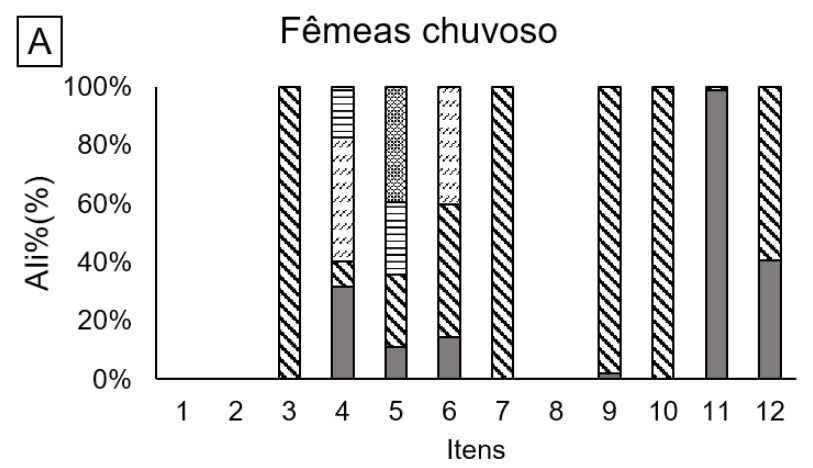

$B$

Fêmeas seco

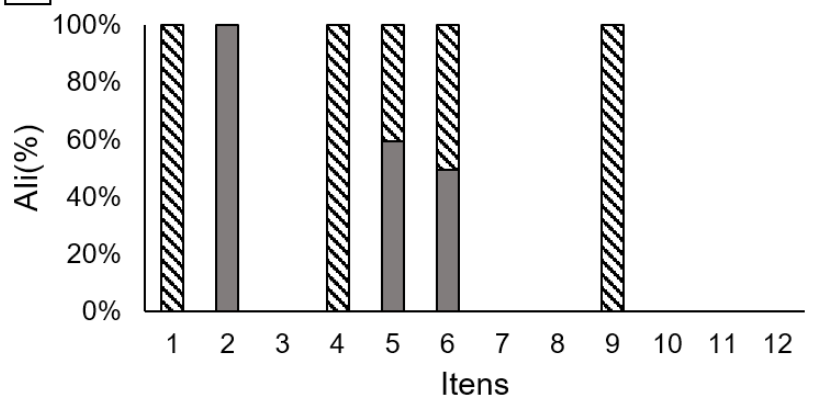

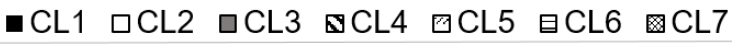

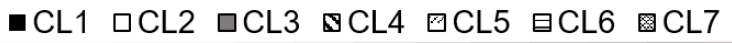
C Machos chuvoso

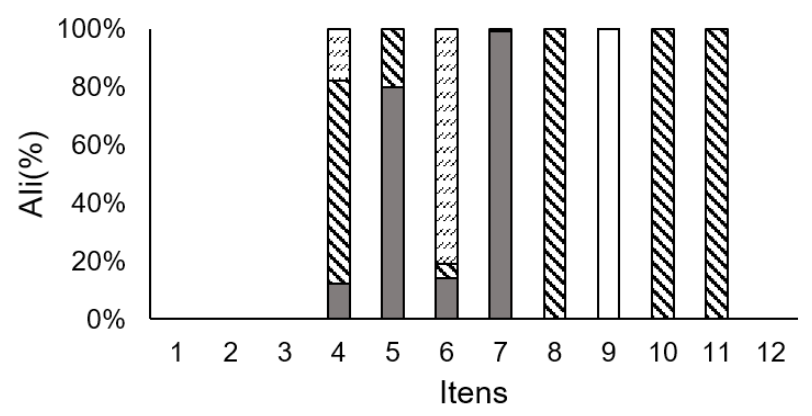

D Machos seco

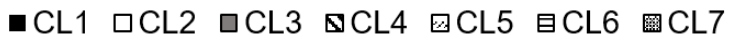

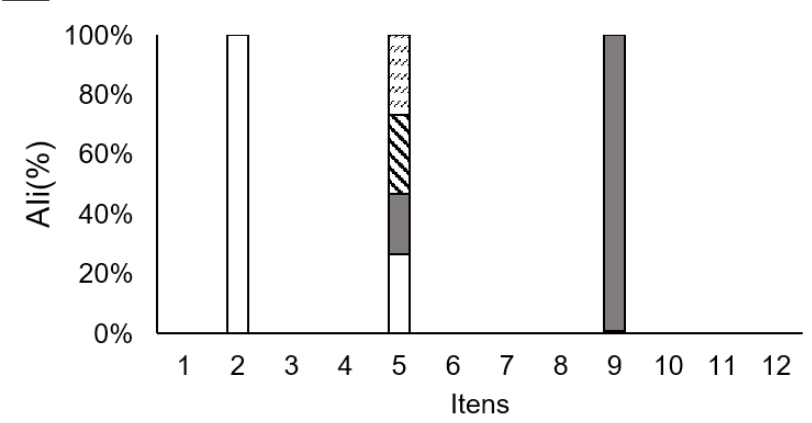

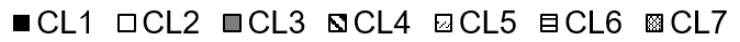

Figura 7 - Variação por classe de tamanho e número de indivíduos relativos e índice de importância alimentar por classe de tamanho de S. parkeri de setembro/2011 a dezembro/2012 capturados na costa Norte do Brasil. Ali = índice de importância alimentar. $F=$ fêmea; $M=$ macho; CL1:<35 cm; CT2: 35 a 40 cm; CL3: 40 a 45 cm; CL4: 45 a 50 cm; CL: 50 a 55 cm; CL6: 55 a 60 cm e CL7: > 60 cm. A = fêmeas de S.parkeri capturados no período chuvoso; B = fêmeas de S.parkeri capturados no período seco; C = machos de S.parkeri capturados no período chuvoso; e D = machos de S.parkeri capturados no período seco. 1 = Ariidae spp; 2 = Callinectes danae; 3 = Cathorops sp; 4 = Cycloplax pinnotheroides; 5 = fragmentos de crustáceos; 6 = fragmentos de peixes; 7 = Macrodon ancylodon; 8 = Panopeus sp; 9 = Penaeidae spp; 10 = Sciaenidae spp; 11 = Stellifer sp.; 12=Xiphopenaeus kroyeri.

\section{DISCUSSÃO}

As fêmeas e os machos da gurijuba capturadas durante todo o período de estudo, encontravam-se na faixa de tamanho de 40 a $50 \mathrm{~cm}$ e com estágio de maturação gonadal imaturas. Esta situação estabelece dúvidas quanto à seletividade atualmente adotada pela frota pesqueira artesanal, tratando-se da captura de indivíduos ainda jovens.

As fêmeas apresentaram maior representa- tividade numérica em comparação aos machos na maioria das classes de tamanho. Segundo a hipótese levantada por Nikolsky (1963), as fêmeas de bagres marinhos ocorrem mais frequentemente em plataformas continentais com grande quantidade de matéria orgânica em suspensão, se enquadrando com as características da área de captura deste estudo.

Nas análises de conteúdo estomacal de S. parkeri, foi observado um considerável número de estômagos com sedimentos e vazios. Por se tratar 
de um bagre de hábitos bentônicos (Lasso-Alcalá et al.,2008), a ingestão do sedimento ocorre, geralmente, de forma acidental durante o consumo de uma presa (Burgess, 1989). A presença de estômagos vazios pode estar também relacionada à rápida digestão do alimento (Fonteles - Filho, 2011) somado ao tempo em que as espécies ficam aprisionadas nos anzóis dos espinhéis ou em alguns casos ao regurgitamento quando os espécimes foram içados.

A gurijuba apresenta hábito alimentar predominantemente carnívoro, onde a dieta foi representada principalmente por crustáceos. A preferência deste item já foi registrada por outros autores para diversos representantes da família Ariidae (Giarrizzo \& SaintPaul 2008; Mendes \& Barthem, 2010; Ribeiro et al., 2012). A presença destes itens na dieta pode estar relacionada às adaptações que os bagres adquiriram no aparelho bucal, como as suas placas dentígeras rígidas, que permitem a alimentação de materiais rígidos (Ribeiro et al., 2012). Outro fator que contribui para frequência dos crustáceos na dieta é a alta diversidade destes recursos no ambiente, devido ao grande aporte de nutrientes provenientes da desembocadura do rio Amazonas, que favorece a produção de várias espécies de crustáceos dentre outros recursos (Bentes et al., 2012). Essa assertiva denota que esta área é essencialmente rica (Oliveira et al., 2007) e por isso é procurada pelas frotas comerciais pesqueiras, em especial a frota industrial que atua com arrastos de fundos nos bancos camaroeiros de toda a costa (Bentes et al., 2012).

O item 'peixes' também esteve presente, em menores proporções, nos indivíduos maiores que 40 $\mathrm{cm}$ de S. parkeri, tanto no período chuvoso quanto no seco. Essas variações na dieta por classe de tamanho mostram uma mudança gradativa da incorporação dos peixes no consumo alimentar mediante o crescimento da espécie, possivelmente pelo aumento da abertura bucal, que garante a captura e a alimentação de presas maiores Ribeiro et al., (2012). Assim, parece provável que S. parkeri apresente ao longo do ciclo de vida, uma variação na dieta condizente com a idade.

No período chuvoso, a dieta de S. parkeri apresentou uma maior amplitude de presas. Callinectes danae, Panopeus sp , Xiphopenaeus kroyeri, Scianidae spp, Stelifer sp, Ariidae spp, Cathorops sp e outros crustáceos e peixes estiveram presentes em maiores proporções do que no período seco. Giarrizzo \& SaintPaul (2008) relatam fatos semelhantes estudando a dieta de Sciades herzbergii. Provavelmente, devido ao aumento do aporte de nutrientes e a carga sedimentar que esta estação promove (Tundisi \& Matsumura, 2008; Esteves, 2011) juntamente com a expansão das marés, correntes costeiras, ondas e ventos constantes na plataforma continental Norte (Aller \& Aller, 1986).
Em função da sazonalidade e sexo, o volume do estômago diferiui significativamente. Estes dois fatores podem estar relacionados ao ciclo reprodutivo da espécie, que segundo Bentes et al. (2012), desovam de novembro a março. A gurijuba e ariídeos em geral, possuem como uma das principais características o cuidado parental dos machos que incubam ovos na boca (Figueiredo \& Menezes, 1980; Moyle \& CechJúnior, 2000), logo, necessitam acumular energia para o período de reprodução.

É evidente que o conhecimento da dieta de um recursos pesqueiros economicamente importantes vem suprir a necessidade da compreensão das relações ecológicas existentes, da disponibilidade de alimento e a influência de outros fatores como os ontogenéticos em Sciades parkeri. Neste estudo, a dieta foi constituída de fontes autóctones do ambiente, principalmente crustáceos que apresentaram frequência constante em todas as estações do ano, classes de tamanho e sexo.

\section{REFERÊNCIAS BIBLIOGRÁFICAS}

Abelha; M. C. F.; Agostinho, A. A; \& Goulart, E. 2001. Plasticidade trófica em peixes de água doce. Maringá, 23 (2). 425-434.

Aller, J.Y. \& Aller, C.R. 1986. General characteristic of benthic faunas of the Amazon inner continental shelf with comparison to the shelf off the Changjiang River, East China Sea. Cont. Shelf Res, 6 (1/2): 291-310.

Araújo A.R. de R. 2001. Dinâmica Populacional e pesca da Gurijuba Arius parkeri (Traill, 1832) (Siluriforme, Ariidae), na costa Atlântica do estado do Amapá. (Dissertação de mestrado). Fortaleza; Universidade Federal do Ceará, Engenharia de Pesca.

Almeida, V.L.L.; Resende, E.K.; Lima, M. S.\& Ferreira, C.J.A.1993. Dieta e atividade alimentar de Prochilodus lineatus (Characiformes, Prochilodontidae) no Pantanal do MirandaAquidauana, Mato Grosso do Sul, Brasil. Rev. Unimar, 15(suppl): 125-141.

Barthem, R.B. \& Fabré, N.N. 2004 Biologia e diversidade dos recursos pesqueiros na Amazônia. In: Rufino, M.L. ( $1^{\text {a }}$ ed.). A pesca e os recursos pesqueiros na Amazônia. Manaus: Ibama/ ProVárzea.p 16-62pp.

Bentes, B.S; Isaac, V.J.; Espirito-Santo, R.V.; Frédou, T.; Almeida M.C.; Mourão, K.R.M. \& Frédou, F.L. 2012 Multidisciplinary approach to identification of Fishery production systems on the northern coast of Brazil. Biota. Neotrop. 12(1): 81-92.

Burgess, W. E. 1989. Family Ariidae. An Atlas of 
freshwater and marine catfishes: a preliminary survey of the Siluriformes. New York: T.F.H. Publications. 159-170 pp.

Canam, B. \& Gurgel, H. C. B. 2002 Ritmicidade da alimentação e dieta de Metynnis roosevelti Eigenmann (Characidae, Myleinae) da Lagoa do Jiqui, Parnamirim, Rio Grande do Norte, Brasil. Revta bras. Zool.19(2): 309 - 630.

Cervigón, F., R. Cipriani, W. Fischer, L. Garibaldi, M. Hendrickx, A.J. Lemus, R. Márquez, J.M. Poutiers, G. Robaina \& B. Rodríguez, 1992. Fochas FAO de identiFocación de especies para los Fones de la pesca. Guia de campo de las especies comercialesmarinas y de aquas salobres de la costa septentrional de Sur America.FAO, Rome.. Preparado con el Fonanciamento de la Comision de Comunidades Europeas y de NORAD 513 p.

Esteves, F.A. 2011. Fundamentos de Limnologia. $3^{a}$ edição. Rio de Janeiro, Interciência, 826p.

Figueiredo, J.L. \& Menezes, N.A. 1980 Manual de peixes marinhos do sudeste do Brasil. II. Teleostei (3). São Paulo. Museu de Zoologia da Universidade de São Paulo, 110p.

Fonteles-Filho, A.A. 2011 Oceanografia, biologia, dinâmica populacional de recurso pesqueiros. $1^{\circ}$ Edição. Expressão Gráfica e Editora. 464p.

Giarrizzo, T. \& Saint-Paul, U. 2008. Ontogenetic and seasonal shifts in the diet of the pemecou sea catfish Sciades herzbergii (Siluriformes: Ariidae), from a macrotidal mangrove creek in the Curuçá estuary, Northern Brazil. Rev. Biol. trop. 56 (2): 161-873.

Hammerschlag, N.; Ovando, D.; Serafy, J.E.2010. Seasonal diet and feeding habits of juvenile Fishes foraging along a subtropical marine ecotone. Aquat. Biol. 9: 279-290p.

Hynes, H.B.N. 1950. The food of fresh-water sticklebacks (Gasterosteus aculeatus e Pygosteus pungitius), with a review of methods used in studies of the food of fishes. Jour. Anim. Ecol., 19 (1): 36-57.

Hyslop, E.J. 1980. Stomach content analysis: a review of methods and their applications. Jour. Fish Biol. 17:411-429.

Instituto Nacional de Meteorologia (INMET). Instituto Nacional de Meteorologia. Estação de Macapá. Disponível em: < http://www.inmet.gov.br/portal/ index.php? $r=$ home/page\&page=rede_estacoes_ conv_graf > Acesso: 20 fev.2015.

Isaac, V.J. 2006. Explotação e manejo dos recursos pesqueiros do litoral amazônico: um desafio para o futuro. Ciênc Cult., 58(3): 33-36.

International Union for Conservation of Nature and Natural Resources (IUCN). 2014. Versão 2014.3. Disponível em: <http://www.iucnredlist.org/ details/155018/0>. Acesso em: 11 mar.2015.

Lasso-Alcalá, O.M.; Lasso, C. A \& Rodríguez, J. 2008. Comunidad de peces demersales del sector suroriental del golfo de Paria, Venezuela. Memoria de la Mem. Fund. La Salle Cienc. Nat.170: 99-124.

Layman, C.A; Quattrocchi, J. P,Peyer, C. M.; Allgeier, J. E. and Suding K. 2007. Niche width collapse in a resilient top predator following ecosystem fragmentation. Eco. Lett. 10: 937-944.

Lima-Junior. E.S.\& Goitein, R. 2001. A new method for the analysis of Fish stomach contents. Maringá. Acta Sci. 23 (2). 421-424.

Lukoschek, V.; McCormick M.I. 2001. Ontogeny of diet changes in a tropical benthic carnivorous Fish, Parupeneus barberinus (Mullidae): Relationship between foraging behaviour, habitat use, jaw size, and prey selection. Mar. Biol.138 (6): 1099-1113.

Marceniuk, A.P. 2005. Chave para Identificação das Espécies de Bagres Marinhos (Siluriformes, Ariidae) da Costa Brasileira. Artigo científico do museu de zoologia da Universidade de São Paulo. Bol.Inst. Pesca. São Paulo.

Melo, G. A. S. 1996. Manual de identificação dos Brachyura (caranguejos e siris) do litoral brasileiro - São Paulo: Plêiade/ FAPESP, 604p.

Mendes F.L.S. \& Barthem, R.B. 2010 Hábitos alimentares de bagres marinhos (Siluriformes: Ariidae) do estuário Amazônico. Amazônia: Ci. \& Desenv. 5 (10). 153-166.

Ministério Do Meio Ambiente (MMA). 2006. Programa REVIZEE. Avaliação do potencial sustentável de recursos vivos na Zona Econômica Exclusiva: Relatório executivo. MMA, Secretaria de Qualidade Ambiental. Brasília. 280p.

Ministério Do Meio Ambiente (MMA). 2008. Macro diagnóstico da Zona Costeira e Marinha do Brasil. Brasília. 242p.

Ministério da Pesca e Aquicultura (MPA). 2006. Monitoramento da Atividade Pesqueira no Litoral do Brasil - Relatório Técnico Final. (Convênio SEAP/PROZEE/IBAMA N0109/2004). Brasília - DF. 328p. Acessado em 09/02/2016. Disponível em: http://www.mpa.gov.br/files/docs/ Informacoes_e_Estatisticas/Monitoramento-daAtividade-Pesqueira-no-Litoral-do-Brasil.pdf

Moyle P.B. \& CECH JUNIOR J.J. 2000. Fishes: an introduction to ichthyology. $5^{a}$ edição .Upper Saddle River: Prentice-Hall. 590p.

Nascimento, R.C.; Folho, M. Santo S, F.J. F.\& Holanda, F.C.A.F. 2002 Distribuição e abundância das principais espécies de bagres estuarinos/marinhos (Ariidae) na plataforma continental Norte do Brasil (PARÁ-AMAPÁ). Bol. Téc. Cient. CEPNOR, 2 (1). 129-145.

Nikolsky G.V. 1963. The Ecology of Fishes. London, 
Academic. 352p.

Oliveira, D. M; Frédou, T.\& Lucena, T. 2007. A pesca no Estuário Amazônico: uma análise uni e multivariada. Bol. Mus. Para. Emílio Goeld. Ciencias Naturais. Belém 2 (2) 11-21.

Pinheiro, L. A. \& Frédou, F. L. 2004. Caracterização geral da pesca industrial desembarcada no Estado do Pará. Rev. Cient. da UFPA. 4. 1-16.

Ribeiro, E.B.; Almeida, Z.S. \& Carvalho-Neta, R.N.F. 2012. Hábito alimentar do bagre Sciades herzbergii (Siluriformes, Ariidae) da llha dos Caranguejos, Maranhão, Brasil. Arq. Bras. Med. Vet. Zootec. 64 (6). 1761-1765.

STATSOFT, INC. 2007 Dada analysis software system, version 7. URL: <www.statsoft.com>

Stephens, D.W..\& Krebs, J.R.1986.Foraging theory. Princeton University Press. 247 p.
Tundisi, J.G. \& Matsumura-Tundisi, T. Limnologia, São Paulo: Oficina de Textos, 2008, 632 p.

Vazzoler, A.E.A.M. 1996 Biologia da Reprodução de Teleósteos: Teoria e Prática. Maringá, PR: Eduem, $169 \mathrm{p}$.

Viana, A.P.; Lucena Frédou, L.; Torres M. D.; \& Bordalo, A.O. 2010. Fish fauna as an indicator of environmental quality in an urbanised region of the Amazon estuary. Jour. Fish Biol. 76: 467-486.v

Wolff, M.; Koch, V.\& Isaac, V. 2000. A Trophic flow model of the Caeté mangrove estuary (north Brazil) with considerations for the sustainable use of its resources. Est. Coast. Shelf Sci., 50: 789-803.

Submetido: Abril/2014 Revisado: Outubro/2015 Aceito: Fevereiro/2016 